\title{
Dictynna
}

Dictynna

Revue de poétique latine

1 | 2004

Varia

\section{Tradizione bucolica e programma poetico in Calpurnio Siculo}

\section{Enrico Magnelli}

\section{OpenEdition}

Journals

\section{Edizione digitale}

URL: http://journals.openedition.org/dictynna/168

DOI: $10.4000 /$ dictynna. 168

ISSN: 1765-3142

\section{Edizione cartacea}

ISBN: 2-84467-088-1

\section{Notizia bibliografica digitale}

Enrico Magnelli, «Tradizione bucolica e programma poetico in Calpurnio Siculo », Dictynna [En ligne], 1 | 2004, mis en ligne le 15 novembre 2010, consulté le 10 décembre 2020. URL : http:// journals.openedition.org/dictynna/168; DOI : https://doi.org/10.4000/dictynna.168

Questo documento è stato generato automaticamente il 10 décembre 2020.

\section{(c) (i) (9)}

Les contenus des la revue Dictynna sont mis à disposition selon les termes de la Licence Creative Commons Attribution - Pas d'Utilisation Commerciale - Pas de Modification 4.0 International. 


\title{
Tradizione bucolica e programma poetico in Calpurnio Siculo
}

\author{
Enrico Magnelli
}

1 Nella seconda metà del secolo scorso, il breve corpus poetico di Calpurnio Siculo ha beneficiato di notevoli cure scientifiche: oltre a tre nuove edizioni critiche (Raoul Verdière, Dietmar Korzeniewski, Jacqueline Amat ${ }^{1}$ ), abbiamo avuto studi importanti sulla sua tradizione manoscritta, sulla sua lingua, sulle sue caratteristiche letterarie e sul suo rapporto con l'ideologia imperiale, nonché numerosi articoli su ciascuna egloga e un serrato dibattito sulla sua cronologia (un problema che non è il caso di affrontare in questa sede: per il momento posso solo dire che anch'io, come molti altri studiosi, ritengo preferibile la datazione tradizionale all'epoca di Nerone ${ }^{2}$ ). Non abbiamo tuttavia uno studio che analizzi l'uso da parte di Calpurnio della tradizione bucolica, non come un mero componente della sua tecnica letteraria, bensì come un elemento costitutivo della sua poetica. È appunto questo che mi propongo di fare.

Ben nota è la grande varietà tematica di Calpurnio, molto maggiore che nelle bucoliche virgiliane. Nella seconda egloga, il nostro poeta mette in scena un giardiniere, figura estranea al mondo pastorale, che descrive la sua arte con numerose riprese dalle Georgiche $e^{3}$; sulle Georgiche è modellata la quinta, costituita dai dettagliati precetti del vecchio Micone al suo giovane figlio Canto sull'allevamento del bestiame ${ }^{4}$; nella terza abbiamo una scena di gelosia tratta dal quattordicesimo idillio di Teocrito ${ }^{5}$; la settima è il resoconto degli spettacoli offerti dall'imperatore al popolo di Roma, e probabilmente Calpurnio si è ispirato al quindicesimo idillio teocriteo (le Siracusane) ${ }^{6}$. Proprio in base a tale varietà di temi non pastorali si è detto che in epoca neroniana «i limiti del genere bucolico sono completamente scardinati $»^{7}$. Questo può esser vero, ma un simile scardinamento non si è verificato né in modo casuale, né per un semplice desiderio di sperimentalismo. In effetti, Calpurnio accoglie nella sua opera molti elementi estranei alla poesia bucolica tradizionale, ma non ricava questi elementi da un grande varietà di fonti: tranne che nella terza egloga, in cui i motivi virgiliani e teocritei si intrecciano con numerosi riecheggiamenti dell'elegia d'amore (conto di tornare su questo punto in 
altra occasione $)^{8}$, i suoi modelli si riducono essenzialmente a Teocrito e a Virgilio, i suoi due più rilevanti predecessori nell'ambito della poesia pastorale.

3 La prima cosa che dobbiamo osservare è che gli elementi non bucolici sono regolarmente 'bucolicizzati', cioè integrati in un contesto pienamente bucolico'. Così nella terza egloga la trama del quattordicesimo idillio di Teocrito, ossia la gelosia del giovane Tionico per l'etera Cinisca (due persone di città), è trasferita da Calpurnio nel mondo pastorale di personaggi chiamati Iolla, Licida, Fillide; così, nella descrizione degli spettacoli nella settima egloga, lo spettatore non è un cittadino come le Siracusane di Teocrito, bensì il pastore Coridone, rappresentato in un modo che ricorda il Titiro della prima bucolica virgiliana ${ }^{10}$. Lo stesso si verifica nei confronti di Virgilio. La profezia di Fauno nella prima egloga di Calpurnio deriva da quella che il dio proferiva rivolgendosi a Latino in Aen. 7, 96-101 ${ }^{11}$, ma il destinatario non è più un re, bensì $\mathrm{i}$ pastori, «la mia gente », come dice il dio (vos o praecipue nemorum gaudete coloni, / vos populi gaudete mei, vv. 36-37), in un contesto perfettamente pastorale ${ }^{12}$. La quinta egloga è modellata sulle Georgiche, ma parla solo di allevamento (non di agricoltura), e la chiusa, con il monito "rientriamo, si fa sera" (vv. 120-121), è quella tipica delle Bucoliche (su questo torneremo più oltre); nella seconda il giardiniere, di cui si è già menzionata l'alterità rispetto al mondo bucolico, si misura tuttavia con un pastore in un canto amebeo tipicamente pastorale, in presenza di numerosi pastori e con un arbitro chiamato Tirsi. Certo Calpurnio sembra più simile a Teocrito per la sua varietà di temi («cette variété est celle des Idylles de Théocrite plus que de Virgile», come ha giustamente affermato Jacqueline Amat ${ }^{13}$ ); d'altro canto, la coerenza della cornice pastorale è quella di Virgilio direi anzi ancor più accentuata. C'è pur sempre un caso in cui Virgilio lascia quasi completamente da parte il mondo dei pastori, nella quarta egloga (ove l'elemento bucolico si riduce all'invocazione iniziale delle Sicelides Musae, alla menzione di Pan e dell'Arcadia ai vv. 58-59 e al carattere genericamente 'campestre' della descrizione dell'età dell'oro), mentre Calpurnio non ammette alcuna eccezione alla regola.

4 Forse Calpurnio voleva conciliare, nel suo breve libellus bucolico, due importanti caratteristiche dei suoi due illustri predecessori? Può darsi; ma credo che ci sia dell'altro. Per la trasposizione di motivi teocritei non bucolici in un contesto bucolico, il modello fondamentale di Calpurnio era lo stesso Virgilio, che nell'ottava egloga (vv.

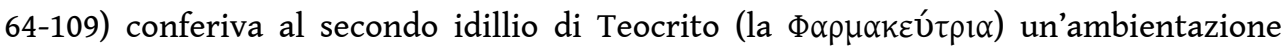
pastorale (i personaggi non si chiamano più Simeta, Testili, Delfi, bensì Dafni, Meris, Amarillide) e, soprattutto, una cornice pastorale (il canto di Alfesibeo) ${ }^{14}$. Questo dato storico-letterario è generalmente ignorato negli studi su Calpurnio, ma, a mio avviso, è tutt'altro che insignificante. Da un lato Calpurnio imita questo procedimento virgiliano nelle egloghe 3 e 7, dall'altro lo estende allo stesso Virgilio, trasponendo nel mondo pastorale elementi dell'Eneide o delle Georgiche nelle egloghe 1, 2 e 5. L'imitazione si fa emulazione; Calpurnio riconosce un aspetto importante della tecnica compositiva di Virgilio, lo sistematizza (mettendo in risalto la sua capacità di fare ciò che faceva il suo illustre predecessore) e lo applica al suo stesso modello (utilizzando così l'opera di Virgilio nello stesso modo in cui Virgilio utilizzava quella di Teocrito). L'innovazione, lungi dal frantumare la tradizione, si rapporta ad essa in termini di confronto e di continuità una continuità non di temi, bensì di poetica e di tecnica letteraria.

5 L'importanza che Calpurnio attribuisce al suo status di poeta bucolico appare chiaramente nella sua autorappresentazione. Quasi tutti gli studiosi affermano 
concordemente che il pastore Coridone, protagonista delle tre egloghe 'politiche' $(1,4$, 7), si deve identificare con l'autore ${ }^{15}$ : è lui che canta le lodi dell'imperatore nella settima egloga, è lui che nella quarta lamenta le difficoltà economiche dei poeti, auspica maggior prosperità sotto il nuovo regime e, soprattutto, stabilisce la sua relazione con la tradizione letteraria precedente :

CO. quod si tu faveas trepido mihi, forsitan illos experiar calamos, here quos mihi doctus Iollas donavit dixitque : " truces haec fistula tauros 60 conciliat nostroque sonat dulcissima Fauno. Tityrus hanc habuit, cecinit qui primus in istis montibus Hyblaea modulabile carmen avena ".

ME. magna petis, Corydon, si Tityrus esse laboras. ille fuit vates sacer et qui posset avena 65 praesonuisse chelyn, blandae cui saepe canenti adlusere ferae, cui substitit advena quercus, quem modo cantantem rutilo spargebat acantho Nais et implicitos comebat pectine crines.

CO. est, fateor, Meliboee, deus : sed nec mihi Phoebus 70 forsitan abnuerit. tu tantum commodus audi : scimus enim, quam te non aspernetur Apollo.

La bibliografia sulla quarta egloga è abbondantissima, e non è il caso di riassumere qui tutte le discussioni nate su questo passo ${ }^{16}$. Ad ogni modo, è evidente che Titiro rappresenta Virgilio, il poeta delle Bucoliche; Coridone-Calpurnio, nel dialogo con Melibeo, suo patrono e giudice letterario (che gli studiosi hanno identificato con Seneca o con altri personaggi influenti dell'epoca $\left.{ }^{17}\right)$, non esita a riconoscerne l'eccellenza, ma al contempo si presenta come il suo erede poetico. Ma perché Coridone? In Virgilio, Coridone è il protagonista della seconda egloga : rusticus es, Corydon, dice a se stesso il pastore virgiliano (ecl. 2, 56), e questa stessa rusticitas è ammessa dal personaggio di Calpurnio, sia nel suo aspetto,

o utinam nobis non rustica vestis inesset ! $\quad(7,79)$, sia nella sua poesia :

nunc mea rusticitas, si non valet arte polita

carminis, at certe valeat pietate probari. $\quad(4,14-15)$.

7 Eleanor Leach, conformemente alla sua interpretazione delle egloghe 'politiche' come espressione della disillusione del poeta e della sua critica del potere imperiale, ritiene che Coridone sia stato scelto a motivo della sua evidente naïveté ${ }^{18}$. Come vari altri studiosi ${ }^{19}$, non mi sento di condividere la sua teoria : ma questa è un'altra storia. Quello che ci interessa rilevare è che il Coridone virgiliano non è solo il rusticus della seconda egloga, ma è anche il celebre cantore della settima, come Melibeo (nome che Calpurnio conferirà al suo patrono, come abbiamo già visto), spettatore della sua gara con Tirsi, afferma nel distico finale $(7,69-70)$ :

haec memini, et victum frustra contendere Thyrsim.

ex illo Corydon Corydon est tempore nobis ${ }^{20}$.

Non sappiamo se l'interpretazione di Coridone come allegoria di Virgilio, attestata nei commentatori tardoantichi, fosse già nota a Calpurnio, come Volker Langholf ha sostenuto nel suo innovativo studio ${ }^{21}$; ma certo, per la scelta del nome Coridone, la settima egloga virgiliana non ha avuto meno importanza della seconda. CalpurnioCoridone non pretende di essere un vates sacer $(4,65)$, un deus $(4,70)$ come Virgilio, e tuttavia, entro i limiti della sua rusticitas, cioè della poesia bucolica ${ }^{22}$, non esita ad 
affermare la sua preminenza ed a proporsi come il legittimo erede del suo grande predecessore letterario ${ }^{23}$.

9 L'importanza del genere bucolico è probabilmente ribadita in un altro passo del liber calpurniano. Nella seconda egloga, il pastore Ida e il giardiniere Astaco iniziano il loro canto amebeo dichiarando la loro investitura 'professionale' ad opera delle rispettive divinità tutelari $(2,28-35)$ :

ID. me Silvanus amat, dociles mihi donat avenas et mea frondenti circumdat tempora taeda. ille etiam parvo dixit mihi non leve carmen : 30 " iam levis obliqua crescat tibi fistula canna ».

AS. et mihi Flora comas pallenti gramine pingit et matura mihi Pomona sub arbore ludit. " accipe ", dixerunt Nymphae, "puer, accipe fontes: iam potes irriguos nutrire canalibus hortos ». 35

Questo passo è ricco di immagini e di vocaboli fortemente sospetti di nascondere un significato poetologico. Davis osserva en passant che « the vocabulary chosen by Astacus is suggestive of song ", menzionando il fons della montagna delle Muse e i canales del flauto di Coridone nella quarta egloga calpurniana (canales / et preme, qui dignas cecinerunt consule silvas, 4, 76-77) ${ }^{24}$. Io penserei piuttosto a due passi virgiliani, ecl. 3, 111 claudite iam rivos, pueri : sat prata biberunt, ove la metafora poetologica è ben chiara, e 6, 69 hos tibi dant calamos, en accipe, Musae, che Calpurnio ha probabilmente ripreso al v. 34 (dall'investitura del divinus poeta Gallo da parte di Lino e delle Muse all'investitura del poeta-giardiniere Astaco da parte delle più umili Ninfe, divinità tradizionalmente bucoliche ${ }^{25}$ ). Ma questo rimane comunque ipotetico. Meno ipotetico, invece, sembra l'uso metaforico di levis al v. 31. È ben vero che negli studi classici la ricerca di significati poetologici è stata più volte spinta all'eccesso, soprattutto nel caso di levis, $\lambda \varepsilon \pi \tau$ ós ed altri termini provenienti da un campo semantico tradizionalmente associato al callimachismo ${ }^{26}$; ma qui è difficile pensare che Calpurnio, memore del celebre deductum carmen virgiliano (ecl. 6, 5) così come del leve carmen del dio Pan in Ovidio, met. 11, 154, non impiegasse l'aggettivo nella sua accezione stilistica ${ }^{27}$. Così, la marcata contrapposizione tra levis e il non leve carmen del verso precedente, lungi da essere solo un gioco retorico ${ }^{28}$, ha a sua volta un significato ben preciso: questo carmen (parola intenzionalmente ambigua, che designa il 'verso profetico' della divinità ma che può parimenti alludere alla poesia) 'non era di poco conto' non solo perché pronunciato da un dio, ma anche perché riferito ad un genere letterario ormai assurto a piena dignità. Per Calpurnio, la poesia bucolica può essere levis nel suo stile, ma certo non è affatto levis nella sua importanza.

11 La 'specializzazione' di Calpurnio come poeta bucolico e la sua diligenza nel sottolineare l'importanza del genere pastorale possono aiutarci a comprendere meglio la quinta egloga, il cui significato complessivo potrebbe sembrare poco chiaro. L'esordio,

forte Micon senior Canthusque, Miconis alumnus, torrentem patula vitabant ilice solem $\quad(5,1-2)$,

riprende quello della settima egloga virgiliana ( 7,1 forte sub arguta consederat ilice Daphnis); il nome di Micone è ben radicato nella tradizione pastorale (Theoc. 5, 112, Verg. ecl. 3, 10 e 7, 30) ; la chiusa,

plura quidem meminisse velim, nam plura supersunt :

sed iam sera dies cadit et iam sole fugato

frigidus aestivas compellit Noctifer horas

$(119-121)$ 
è tipicamente bucolica (cfr. Verg. ecl. 1, 82-83 et iam summa procul villarum culmina fumant / maioresque cadunt altis de montibus umbrae ; 6, 85-86 cogere donec ovis stabulis numerumque referre / iussit et invito processit Vesper Olympo ; 10, 75-77 surgamus; solet esse gravis cantantibus umbra; / iuniperi gravis umbra; nocent et frugibus umbrae. / ite domum saturae, venit Hesperus, ite capellae) ${ }^{29}$. Nondimeno, lo stile didascalico e l'evidente imitazione delle Georgiche, di cui già abbiamo parlato, hanno fatto sì che questa egloga sia stata interpretata da alcuni studiosi come « una pausa e una innovazione " nella produzione del poeta, un primo tentativo di coltivare un diverso genere letterario ${ }^{30}$. Io non lo credo affatto. A mio avviso, alla luce della poetica di Calpurnio si può proporre un'interpretazione esattamente opposta : il poeta non vuole trasformare in georgica la bucolica, bensì 'bucolicizzare' la georgica, riaffermando così la sua fedeltà al genere ${ }^{31} \mathrm{e}$ miniaturizzando la poesia didascalica nel contesto del suo mondo pastorale ${ }^{32}$. La conclusione dell'egloga pare un indizio significativo : "rimangono molti altri precetti », vale a dire "potrei scrivere un'opera come le Georgiche, ma preferisco contenere la mia poesia entro i limiti delle Bucoliche, in cui ogni attività si arresta al calar del sole ».

Da un punto di vista storico-letterario, è perfettamente comprensibile che proprio nell'età di Nerone (se si accetta, come si è detto all'inizio, la cronologia tradizionale) si sia verificata una forte ripresa del genere bucolico, che da un lato soddisfaceva il gusto dell'epoca per una poesia di evasione ${ }^{33}$, dall'altro era adattissima allo sviluppo sistematico di temi encomiastici già attestati in Teocrito e in Virgilio. Ciò può render conto della poetica di Calpurnio, che si specializza nella poesia bucolica e ne ribadisce l'importanza e la dignità, ma che si sforza altresì di temperare le innovazioni mediante una costante attenzione alla salvaguardia della cornice pastorale, evitando che il genere perda la sua identità. Questa non è solo una prova della sua conoscenza dei meccanismi operanti nell'ambito del genere bucolico : è una precisa scelta di poetica. Io non sono il divino Virgilio, afferma Calpurnio, né voglio fare tutto ciò che ha fatto lui ; io sono solo bucolico. Ma nel mio ristretto campo, nel campo di un genere 'inferiore' come la bucolica, ho imparato a possedere a fondo la tradizione letteraria e ad impiegare i 'ferri del mestiere' del mio predecessore con un rigore ancor più stretto del suo. Io sono un Virgilio 'specializzato', e mi va benissimo così. Nec te paeniteat pecoris, senza alcun dubbio ${ }^{34}$.

\section{NOTE}

1. R. Verdière, T. Calpurnii Siculi De laude Pisonis et Bucolica, Berchem-Bruxelles 1954; D. Korzeniewski, Hirtengedichte aus neronischer Zeit, Darmstadt 1971; J. Amat, Calpurnius Siculus, Bucoliques. Pseudo-Calpurnius, Éloge de Pison, Paris 1991. Abbiamo inoltre l'edizione commentata di ecl. 7 ad opera di L. Di Salvo (Bologna 1990) e il commento a ecl. 4 di B. Schröder (Carmina non quae nemorale resultent, Frankfurt am Main-Bern-New York-Paris 1991). Una nuova edizione critica è annunciata da Maria Assunta Vinchesi nella sua utilissima proecdosis: Calpurnio Siculo. Egloghe, Milano 1996, 55. 
2. La vecchia teoria di una datazione al III secolo è stata riproposta da $\mathrm{E}$. Champlin, The life and times of Calpurnius Siculus, "JRS» 68, 1978, 95-110 (cui fanno seguito History and the date of Calpurnius Siculus, "Philologus»130, 1986, 104-112, e D. Armstrong, Stylistic and the date of Calpurnius Siculus, ibid. 113-136); cfr. le obiezioni di G. B. Townend, Calpurnius Siculus and the Munus Neronis, «JRS » 70, 1980, 166-174, di R. Mayer, Calpurnius Siculus. Technique and date, ibid. 175-176, e di R. Verdière, A quelle époque vécut T. Calpurnius Siculus ?, "CRDAC»12, 1982-83, 125-138. In favore di una datazione tarda si pronunciano ancora E. Courtney, Imitation, chronologie littéraire et Calpurnius Siculus, « REL » 65, 1987, 148-157, B. Baldwin, Better late than early: reflections on the date of Calpurnius Siculus, «ICS » 20, 1995, 157-167, e N. Horsfall, Criteria for the dating of Calpurnius Siculus, « RFIC »125, 1997, 166-196. La più recente e dettagliata difesa della cronologia tradizionale è quella di R. Verdière, Calpurnius, en fin d'analyse..., « Helmantica » 44, 1993, 349-398 ; cfr. anche J. Fugmann, Nero oder Severus Alexander ? Zur Datierung der Eklogen des Calpurnius Siculus, «Philologus » 136, 1992, 202-207.

3. Amat, 12 ; Vinchesi, Calpurnio Siculo..., 35-36.

4. Amat, 45 ; Vinchesi, Calpurnio Siculo..., 39-40 ; vd. anche infra, n. 27.

5. R. Verdière, La bucolique post-virgilienne, « Eos » 56, 1966, 169.

6. Amat, xxxi.

7. A. Perutelli, Natura selvatica e genere bucolico, "ASNP» 6, 1976, 782 (uno studio tuttora fondamentale sull'evoluzione della poesia bucolica a Roma).

8. Vd. P. J. Davis, Structure and meaning in the Eclogues of Calpurnius Siculus, « Ramus »16, 1987, 35, e soprattutto la dettagliata analisi di M.A. Vinchesi, La terza ecloga di Calpurnio Siculo fra tradizione bucolica e tradizione elegiaca, « Prometheus » 17, 1991, 259-276.

9. Come ha giustamente osservato M. von Albrecht, Geschichte der römischen Literatur II, BernMünchen 1992, 784, « so erweitert Calpurnius die bukolische Gattung doch ohne ihren Rahmen zu springen $»$.

10. Vd. p. es. Di Salvo, 42 (ma l'analogia è cosa nota).

11. Come molti studiosi hanno ben sottolineato. B. Martin, Calpurnius Siculus" "new " aurea aetas, «AClass» 39, 1996, 21, ricorda anche i precetti di Fauno al re Numa in Ov. fast. 4, 649-670: ma lì non si

tratta di una profezia, e il legame con Calpurnio pare assai tenue.

12. Vinchesi, Calpurnio Siculo..., 14; cfr. anche N.W. Slater, Calpurnius and the anxiety of Vergilian influence: Eclogue I, « Syllclass » 5, 1994, 71-78.

13. Amat, xxxvi; cfr. anche P. Esposito, Per la storia della ricezione di Virgilio bucolico: l'ecloga IV di Calpurnio Siculo, « Orpheus »17, 1996, 29.

14. Il primo verso di questa egloga è sintomatico: pastorum Musam Damonis et Alphoesiboei. « Pastorum, as if to assert the pastoral character of the poem as a whole in anticipation of the reader's response to the unpastoral Muse of Alphoesiboeus " (Clausen ad loc.); ma ad essere estraneo alla bucolica non è il canto di Alfesibeo, bensì il suo modello teocriteo.

15. Fa eccezione Davis, Structure and meaning..., 39 e passim.

16. Basti rinviare al commento di Schröder, 21-29 e 119-131.

17. Tra le varie rassegne delle numerose (e a volte del tutto fuorvianti) ipotesi in merito, particolarmente dettagliata è quella di M.D. Spadaro, Sulle egloghe politiche di Tito Calpurnio Siculo, Catania 1969, 27-28 n. 49.

18. E.W. Leach, Corydon revisited. An interpretation of the political eclogues of Calpurnius Siculus, « Ramus » 2, 1973, 85-87. La sua linea esegetica (ribadita in Neronian pastoral and the world of power, « Ramus » 4, 1975, 204-230) è accolta da Davis, Structure and meaning..., passim.

19. Per esempio Vinchesi, Calpurnio Siculo..., 27 e n. 39; A.T. Fear, Laus Neronis: the seventh eclogue of Calpurnius Siculus, « Prometheus » 20, 1994, 269-277.

20. Un verso particolarmente significativo ove se ne riconosca il modello, ossia [Theoc.] 8, $92 \mathrm{k} \eta \hat{k}$

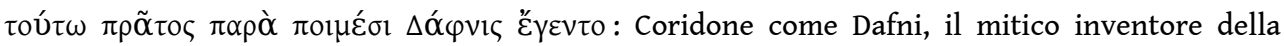


poesia pastorale. Anche alla luce di questo, nutro forti riserve sull'idea di T. K. Hubbard, Calpurnius Siculus and the unbearable weight of tradition, "Helios» 23, 1996, 71, secondo cui i modelli virgiliani servirebbero a Calpurnio per costruire l'immagine di un Coridone « unstable ephebe poet, yearning for recognition, but uncertain of himself ». Quello su cui il Coridone calpurniano deliberatamente si mostra incerto non sono le sue qualità poetiche, bensì i suoi mezzi di sussistenza.

21. V. Langholf, Vergil-Allegorese in den Bucolica des Calpurnius Siculus, « RhM » 133, 1990, 357 e n. 23.

22. Cfr. già Vinchesi, Calpurnio Siculo..., 29 : « il termine sembra alludere non solo alla condizione del personaggio [... ] ma al canto pastorale stesso ».

23. Nella raccolta bucolica virgiliana, Titiro è il protagonista della prima egloga, Coridone della seconda. Forse Calpurnio voleva anche suggerire al suo lettore un'interpretazione cronologica di quest'ordine, Titiro-Virgilio all'inizio della tradizione pastorale latina e Coridone-Calpurnio, suo erede e continuatore, al secondo posto ? Ma questa rimane una pura ipotesi.

24. Structure and meaning..., 52 n. 19.

25. La sostituzione delle Muse con le Ninfe come patrone del canto pastorale, e più in generale la selezione di uno specifico pantheon bucolico,

erano procedimenti già ampiamente esperiti da Teocrito: vd. in particolare Fantuzzi in M. Fantuzzi - R. Hunter, Muse e modelli. La poesia ellenistica da Alessandro Magno ad Augusto, Roma-Bari 2002, 196-203.

26. Per l'àmbito latino, piace ricordare le argute osservazioni di A. Barchiesi, «Gnomon » 69, 1997, 672: « dopo tutto quello che si è scritto in anni recenti sul callimachismo autoriflesso della poesia romana, è da prevedere un certo effetto di saturazione: qualcuno vorrà certo proporre una sospensiva, almeno temporanea, di studi su 'magro' e 'grasso', gigantomachie, navicelle e fiumi in piena » (anche se, come osserva di seguito lo stesso Barchiesi, « una reazione diametrale, oltre che ingenerosa, sarebbe dannosa: c'è il rischio di gettar via il bambino insieme all'acqua sporca dell'Eufrate »).

27. Questo è stato opportunamente sottolineato da K.-H. Stanzel, Bukolische Dreiecksverhältnisse. Zu Calpurnius' und Nemesians zweiter Ekloge, «WJA» 15, 1989, 190 e n. 17 («Wenn Silvanus die fistula levis nennt, dann liegt darin neben dem Gegensatz zu non leve carmen eine Stilbestimmung der Bukolik»).

28. Come afferma Verdière ad loc. : «C. eût pu, par respect pour le dieu, mettre un peu moins d'humour à écrire non leve carmen levis... fistula ».

29. Tutti questi elementi sono stati ben sottolineati dai commentatori.

30. Lo ha sostenuto soprattutto M. Marchiò, Un componimento georgico sulle orme di Virgilio : l'egloga V di Calpurnio Siculo, «GIF »10, 1957, 301-314; i dubbi di R. Verdière, Le genre bucolique à l'époque de Néron: les «Bucolica» de T. Calpurnius Siculus et les "Carmina Einsiedlensia ». État de la question et prospectives, «ANRW » II 32.3, 1985, 1854-55, sono ben fondati. Leach, Neronian pastoral..., 217 afferma giustamente che questa egloga « can hardly be taken for a serious essay into didactic poetry »; ma è difficile accogliere la sua interpretazione pessimistica secondo cui «this anxious recital shows the herdsman's life as a constant dedication of energy to making defenses against nature ", e « Mycon reveals a world where the prospects of youth lead only to weary old age " (l'insistenza di Calpurnio sui verba... tremulis titubantia labris del vecchio Micone al v. 6 sembra nient'altro che un tocco di realismo descrittivo, conforme del resto al gusto del poeta: vd. in proposito E. Di Lorenzo, Aspetti letterari e atteggiamenti espressivi nelle Bucoliche di Calpurnio Siculo, « Misure critiche »18, 1988, 5-21, qui soprattutto 19-20).

31. C. Messina, T. Calpurnio Siculo, Padova 1975, 106, nota giustamente che Calpurnio vuole « dare le dimensioni della bucolica agli insegnamenti georgici»; ma poi anche lui, benché meno decisamente di Marchiò, tende ad interpretare quest'egloga come un distacco dal genere 
autenticamente bucolico. Al contrario : è giustappunto con l'appropriazione di elementi esterni e col loro 'adattamento' alle proprie esigenze che il genere riafferma la propria vitalità.

32. Come mi fa acutamente notare Alfredo Morelli, la sezione delle Georgiche dedicata all'allevamento del bestiame minuto (pecore e capre, come in Calpurnio) era introdotta da Virgilio con versi particolarmente altisonanti e con un'orgogliosa dichiarazione di originalità poetica di stampo callimacheo e lucreziano $(3,286-294)$, a sottolineare la novità di questo argomento anche in relazione al disegno dell'opera ; Calpurnio invece "sembra quasi 'riportare' il tema nell'ambito che sembra più naturale, quello bucolico ». E anche questo avviene forse sulla falsariga di motivi bucolici virgiliani. Lo stesso Morelli osserva (molto giustamente, a mio parere) che tra le varie chiuse delle egloghe virgiliane su cui è modellata quella di Calpurnio, l'analogia più significativa è con l'egloga sesta : «in entrambi i casi abbiamo un canto didascalico che si interrompe all'incedere di Espero, e si interrompe inopportunamente, a malincuore (plura quidem meminisse velim, nam plura supersunt, v. 119 di Calpurnio, malincuore di chi narra; iussit et invito processit Vesper Olympo, v. 86 di Virgilio, malincuore di chi ascolta) ». Se anche il canto di Sileno nella sesta egloga virgiliana può, in virtù del suo carattere cosmogonico ed etiologico, considerarsi come un'esperienza conoscitiva (benché ovviamente priva di qualsiasi precettistica), sarà lecito affermare che « il vecchio Sileno trasmette la sua conoscenza ai due giovani così come Micone la trasmette a Canto in Calpurnio, ma Micone non sceglie i temi così elevati [...] del suo 'predecessore', bensì quelli ristretti e poveri della vita pastorale, reinterpretando Virgilio con Virgilio, e riportando la trattazione sull'allevamento delle capre nel suo più naturale alveo bucolico, una volta che in esso, grazie all'esempio virgiliano, sia stata integrata l'opzione 'didascalica' ».

33. Lo ha messo in luce assai bene Perutelli, Natura selvatica..., 782 ss.

34. È un piacere ringraziare per i loro utili suggerimenti tutti i partecipanti al Colloque di Lille, nonché Gianfranco Agosti, Andrea Cucchiarelli, Claudio De Stefani, Lucia Floridi, Alfredo M. Morelli e Maria-Pace Pieri, che hanno letto in anteprima queste pagine. S'intende che nessuno di loro è vincolato alle mie conclusioni.

\section{AUTORE}

\section{ENRICO MAGNELLI}

Università di Firenze 